\title{
Calcium-dependent blood-brain barrier breakdown by NOX5 limits postreperfusion benefit in stroke
}

\author{
Ana I. Casas, ${ }^{1}$ Pamela W.M. Kleikers, ${ }^{1}$ Eva Geuss, ${ }^{2}$ Friederike Langhauser, ${ }^{3}$ Thure Adler, ${ }^{4,5}$ Dirk H. Busch, ${ }^{5}$ Valerie Gailus-Durner, ${ }^{4}$ \\ Martin Hrabê de Angelis, ${ }^{4,6,7}$ Javier Egea, ${ }^{8}$ Manuela G. Lopez, ${ }^{9}$ Christoph Kleinschnitz, ${ }^{3}$ and Harald H.H.W. Schmidt ${ }^{1}$ \\ 'Department of Pharmacology and Personalised Medicine, CARIM, Maastricht University, Maastricht, The Netherlands. Department of Neurology, University Hospital Würzburg, Würzburg, Germany. \\ ${ }^{3}$ Department of Neurology, University Clinics Essen, Essen, Germany. ${ }^{4}$ Cerman Mouse Clinic, Institute of Experimental Genetics, Helmholtz Zentrum München, German Research Center for Environmental \\ Health, Neuherberg, Germany. Institute for Medical Microbiology, Immunology and Hygiene, Technical University of Munich, Munich, Germany. ${ }^{6}$ Chair of Experimental Genetics, School of Life Science \\ Weihenstephan, Technical University of Munich, Freising, Germany. ${ }^{7}$ Cerman Center for Diabetes Research (DZD), Neuherberg, Germany. ${ }^{8}$ Instituto de Investigación Sanitaria, Servicio de Farmacologia Clínica, \\ Hospital Universitario de la Princesa, Madrid, Spain. ${ }^{9}$ Institute Teofilo Hernando for Drug Discovery, Department of Pharmacology, School of Medicine, Universidad Autónoma de Madrid, Madrid, Spain.
}

\begin{abstract}
Ischemic stroke is a predominant cause of disability worldwide, with thrombolytic or mechanical removal of the occlusion being the only therapeutic option. Reperfusion bears the risk of an acute deleterious calcium-dependent breakdown of the blood-brain barrier. Its mechanism, however, is unknown. Here, we identified type 5 NADPH oxidase (NOX5), a calciumactivated, ROS-forming enzyme, as the missing link. Using a humanized knockin (KI) mouse model and in vitro organotypic cultures, we found that reoxygenation or calcium overload increased brain ROS levels in a NOX5-dependent manner. In vivo, postischemic ROS formation, infarct volume, and functional outcomes were worsened in NOX5-KI mice. Of clinical and therapeutic relevance, in a human blood-barrier model, pharmacological NOX inhibition also prevented acute reoxygenationinduced leakage. Our data support further evaluation of poststroke recanalization in the presence of NOX inhibition for limiting stroke-induced damage.
\end{abstract}

\section{Introduction}

Ischemic stroke represents one of the most frequent causes of death and leading causes of disability worldwide (1). Thrombolytic or mechanical removal of the occlusion is the only therapeutic option (2). In $11 \%$ of the mechanically recanalized patients, however, serious complications occur (3), and thrombolysis using tissue plasminogen activator (rtPA) correlates with an early opening of the bloodbrain barrier (BBB), edema, and hemorrhagic transformation, with excessive ROS production and worse prognosis (4). Importantly, an increase in intracellular calcium represents one of the earliest events during this scenario $(2,5)$. Pharmacological agents that selectively inhibit the activity of ROS-forming enzymes, i.e., NADPH oxidase, are active in poststroke models (6). Antioxidants have been used to restore redox imbalances and improve disease outcomes, although with mostly ineffective results $(6,7)$. We therefore implemented an innovative approach that uses pharmacological agents that selectively inhibit the activity of ROS-forming enzymes (8).

NADPH oxidases are considered a primary and quantitatively relevant source in different ischemic conditions (5). Of particu-

\section{Related Commentary: p. 1530}

Authorship note: AIC and PWMK contributed equally to this work. CK and HHHWS are co-senior authors.

Conflict of interest: The authors have declared that no conflict of interest exists. Copyright: @ 2019 American Society for Clinical Investigation

Submitted: August 16, 2018; Accepted: February 5, 2019.

Reference information: / Clin Invest. 2019;129(4):1772-1778.

https://doi.org/10.1172/JCl124283. lar interest is NADPH oxidase type 5 (NOX5), the only calciumactivated isoform widely expressed in endothelium (7), testis, and WBCs (8). We hypothesized that NOX5 may be the missing link between postreperfusion calcium overload and early BBB opening. Since NOX5 is missing from the mouse genome, we generated a humanized mouse model expressing the human NOX5 gene. To validate its role in acute recanalization, we examined both a pharmacological approach in a human in vitro BBB model and a genetic approach in an in vivo stroke model in mice.

\section{Results and Discussion}

Generation and validation of a humanized NOX5-KI mouse model. In humans, NOX 5 is broadly expressed in vascular endothelium, fibroblasts $(7,9,10)$, testis (9), and spleen monocytes, macrophages $(8,11)$, B cells, and T cells $(12)$. To mimic the physiological human expression pattern of NOX5 in mice, we created a mouse line called NOX5-knockin (NOX5-KI), bearing the human NOX5 gene under control of the Tie2 promoter (Figure 1, A and B). The Tie2 promoter physiologically regulates endothelial and hematopoietic gene expression in human cells $(13,14)$. Therefore, we expected our transgenic mouse model to mimic physiological human NOX5 expression.

Tissues from WT mice did not show any detectable NOX5 gene expression, while significant expression was detected in all KI mice (Figure 1C), primarily in lung, kidney, brain, aorta, and WBCs (monocytes, lymphocytes, T and B cells; Supplemental Figure 1; supplemental material available online with this article; https://doi.org/10.1172/JCI124283DS1). Thus, our Tie2NOX5-KI mouse model is ideally humanized with respect to the 
A

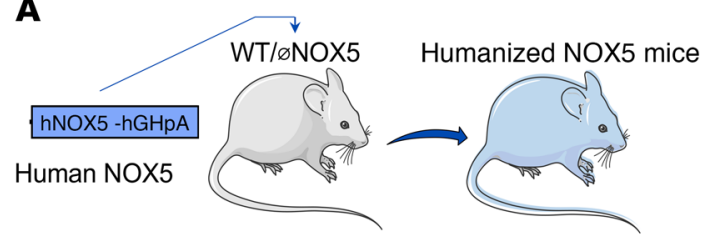

B
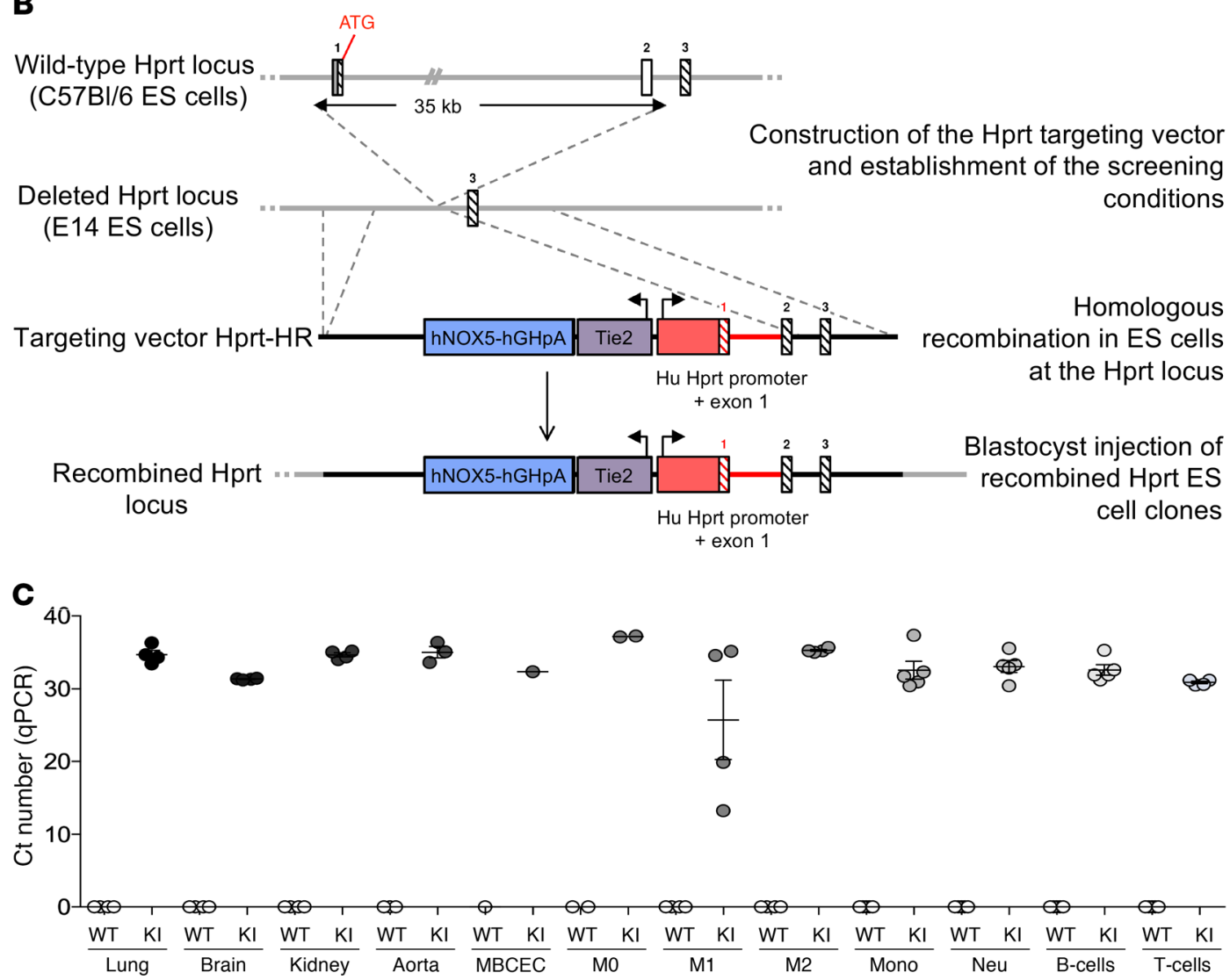

Figure 1. Generation and validation of the NOX5-KI mouse. (A) Representative schematic of the humanized NOX5 mice. NOX5 gene located on the

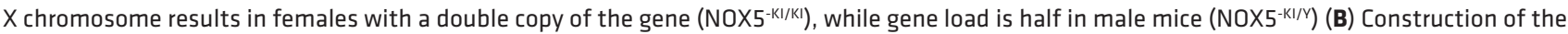
humanized NOX5-KI mice. First, WT and deleted Hprt constructs are shown. The targeting vector to insert NOX5 (human NOX5 cDNA, blue box) was coupled to the human Hprt promoter (red box) and exons 1 and 2. The human Hprt promoter is under control of the Tie2 gene (purple box) to create NOX5 expression. Hatched black and red boxes represent murine and human Hprt exons, respectively. Solid line represents intronic sequences. (C) NOX5 gene expression was measured by qPCR (Ct) in different organs (lung, brain, kidney, aorta, and mouse brain capillary endothelial cells [MBCEC]); macrophages from bone marrow (undifferentiated [MO], inflammatory [M1], and active [M2]); and hematopoietic cells isolated from spleen (monocytes [Mono], neutrophils [Neu], B cells, and T cells). Tissues from WT mice did not show NOX5 gene expression, while significant expression was detected in NOX5-KI mice. Ct values of $n=4$ are shown.

physiological expression patterns in humans, i.e., endothelial cells and WBC. Of note, WBCs are an independent predictor of outcome after ischemic stroke (15). Leukocyte counts may independently predict the risk of suffering from an ischemic event (16). Importantly, the leukocyte proportions in NOX5-KI mice were indistinguishable from those in WT mice, indicating no major phenotype change (Supplemental Table 1 and Supplemental Table 2). Subgroup analysis revealed only a single substantial observation, an increase in the $\mathrm{CD} 4^{+}$to $\mathrm{CD} 8^{+} \mathrm{T}$ cell ratio in females. Thus, any phenotype in our NOX5-KI mouse model will be most likely of endothelial rather than immune cell origin. No phenotype was observed with respect to life span, cognition, or neuromotor function.
In vitro, NOX5 causes acute, calcium-dependent postreoxygenation ROS formation. With respect to ischemic stroke, delayed induction of NOX4 was identified as a relevant source of ROS (5, 17), but neither NOX1 nor NOX2 was identified as such (18), and the role of NOX5 is unclear. To examine whether NOX5 is relevant for ROS formation upon cerebral reoxygenation, we first studied in vitro organotypic hippocampal cultures (OHCs) from NOX5-KI and WT mice. To detect ROS species as broadly as possible, including secondary reactions and not differentiating among superoxide, hydrogen peroxide, hydroxyl, or possibly peroxynitrite, we chose the dihydroethidium (DHE) method, as published previously $(17,19)$. Fifteen minutes of oxygen and glucose deprivation (OGD) (Figure 2A) resulted, unlike with NOX4, in an acute NOX5- 

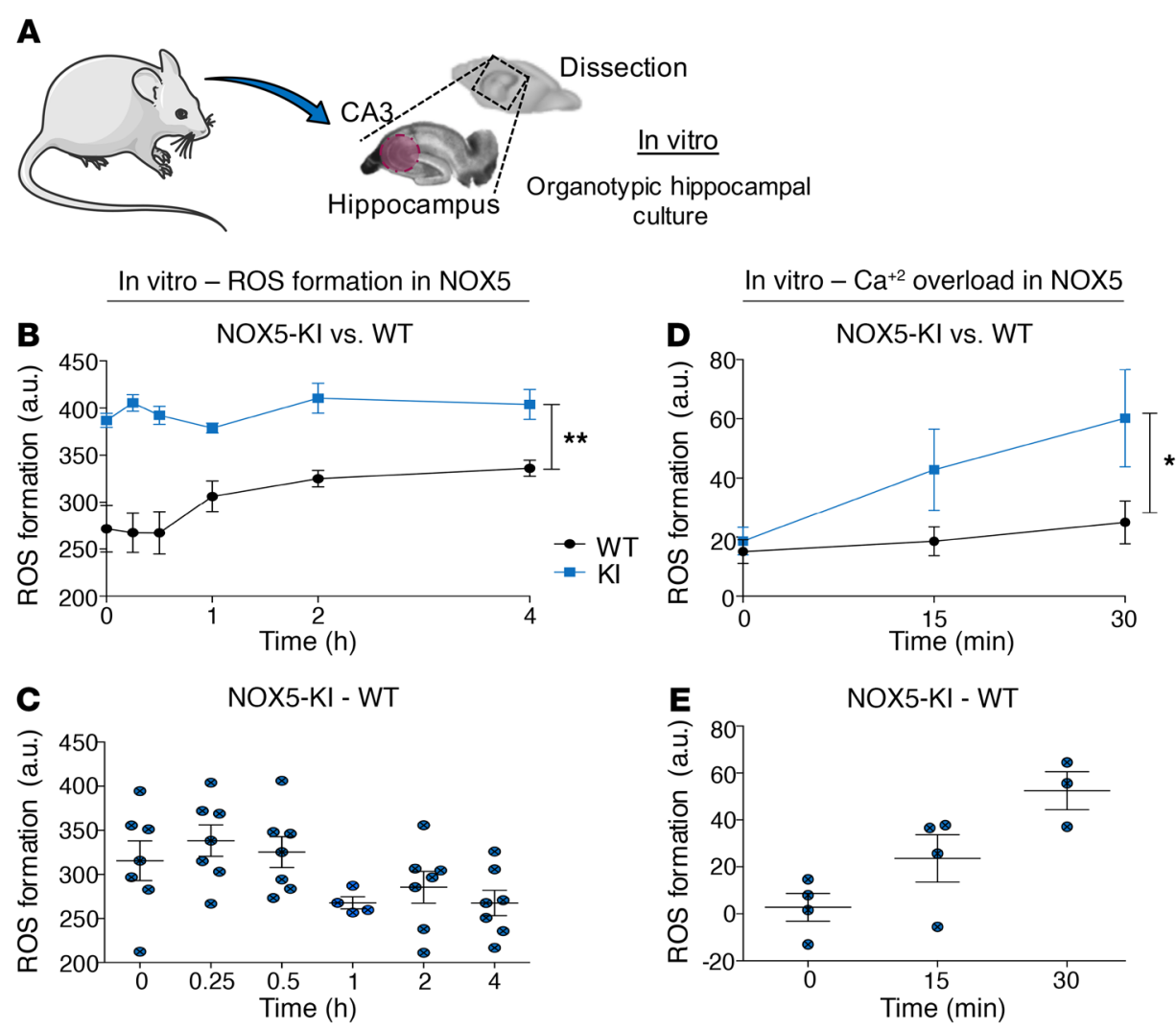

Figure 2. Increased in vitro ROS production is related to $\mathrm{Ca}^{2+}$ overload and NOX5. (A) In vitro, mouse $\mathrm{OHCS}$ were subjected to OGD, followed by the reoxygenation period. (B) ROS production measured after OCD in OHCs was higher in NOX5-KI mice (blue, $n=6$ ) versus NOX5 WT mice (black, $n=6$ ), starting directly from the OCD period until 4 hours after OCD. ${ }^{* *} P<0.01$, Bonferroni's multiple comparison test. (C) Difference in ROS production between NOX5-KI (blue, $n=6$ ) and WT (black, $n=6$ ) mice showed a NOX5-dependent effect within the first hour after $\operatorname{OCD}(0,0.25,0.5$, and 1 hour). (D) Addition of the ionophore A23187 (10 $\mu \mathrm{M})$ showed a $\mathrm{Ca}^{2+}$-dependent increase in ROS formation in NOX5-KI mice (blue, $n=3$, Bonferroni's multiple comparison test), while no changes were detected in WT mice (black, $n=3$ ). ${ }^{*} P<0.05$. (E) Difference in ROS production between NOX5-KI (blue, $n=3$ ) and WT (black, $n=3$ ) mice showed a direct link between $\mathrm{Ca}^{2+}$ overload and NOX5 induction at 15 and 30 minutes after A23187. dependent ROS production within the first 30 minutes that was not present in WT mice (Figure 2, B and C). At later time points, ROS formation was unchanged, presumably due to the known, delayed induction of NOX4 (20). To confirm that the early spike in ROS was indeed due to calcium-induced activation of NOX5, we reexamined ROS generation in OHCs from NOX5-KI and WT mice in the absence or presence of the calcium ionophore A23187 $(10 \mu \mathrm{M})$, but without OGD. Indeed, we observed the same acute ROS surge as seen after OGD and in a strictly NOX5-dependent manner (Figure 2, D and E). Thus, NOX5-dependent ROS formation is involved in early stages after ischemia via a $\mathrm{Ca}^{2+}$-dependent mechanism.

In vivo, ROS formation and BBB breakdown are NOX 5 dependent and worsen neurological outcome. To examine the role of NOX5 in a stroke in vivo, we performed a 1-hour transient occlusion of the middle cerebral artery (tMCAO) on adult mice, followed by 24 hours of reperfusion (Figure 3A) for histological and neurological assessment. Reperfusion injury leads to an abnormally permeable capillary bed, resulting in a disruption of the BBB (4). BBB leakage was significantly increased in brains from NOX5-KI mice when compared with their WT littermates, indicating also here a key role for NOX5 in poststroke BBB integrity (Figure 3B). To examine the mechanistic link between this observation and NOX5 activity, we measured ROS formation in NOX5-KI and WT mice. Again, ROS generation was significantly increased in poststroke NOX5-KI mice compared with controls (Figure 3C). Thus, poststroke NOX5-dependent impairment of the BBB is related to enhanced in vivo ROS formation.

To further evaluate, 24 hours after stroke, whether NOX5dependent ROS formation and impairment of the BBB were linked to a worsened outcome, we assessed infarct sizes and, as a clinical translational parameter, neuromotor function. Importantly, poststroke NOX5-KI mice, when compared with WT mice, showed a significant increase in infarct volume (Figure 3D) and worsened Bederson score and grip tests (Figure 3, E and F; see Supplemental Methods). Thus, acute postreoxygenation $\mathrm{Ca}^{2+}$-dependent ROS formation, BBB breakdown, increased infarct size, and worsened neuromotor outcome are mechanistically linked to NOX5.

Blood pressure does not contribute as a confounder to the NOX5KI phenotype in stroke. Conceivably, an endothelial NOX5-KI may lead to a systemic constitutively elevated endothelial ROS formation linked to increased blood pressure. Hypertension can weaken brain arteries and indirectly worsen stroke outcome (21), which may result in possible overestimation of direct NOX5 impact. We therefore also assessed blood pressure over 24 hours using telemetric devices. Neither systolic, diastolic, nor mean arterial pressure (Supplemental Figure 2, A-C) were different between WT and NOX5-KI mice, suggesting that no systemic hypertensive phenotype confounded the worsened postreperfusion in vivo outcome in NOX5-KI mice.

The role for NOX5-KI in ischemia reperfusion is specific to the brain. In principle, knocking in NOX5 in the vascular endothelium may worsen outcomes in many ischemia-reperfusion (IR) scenarios in different organs and thus play no brain-specific role. Indeed, for NOX1 and NOX2, such roles have been described in myocardial infarction (MI) (22), retinopathy (23), or diabetic nephropathy $(24,25)$. Moreover, NOX5 is upregulated in human blood vessels after MI (26). We therefore investigated in vivo 3 different relevant ischemic-disease conditions: hindlimb ischemia (HL, peripheral artery disease), MI, and IR of the heart. 
A

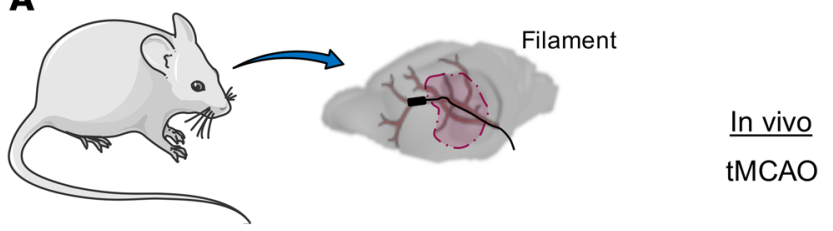

In vivo - Evans blue \& ROS formation
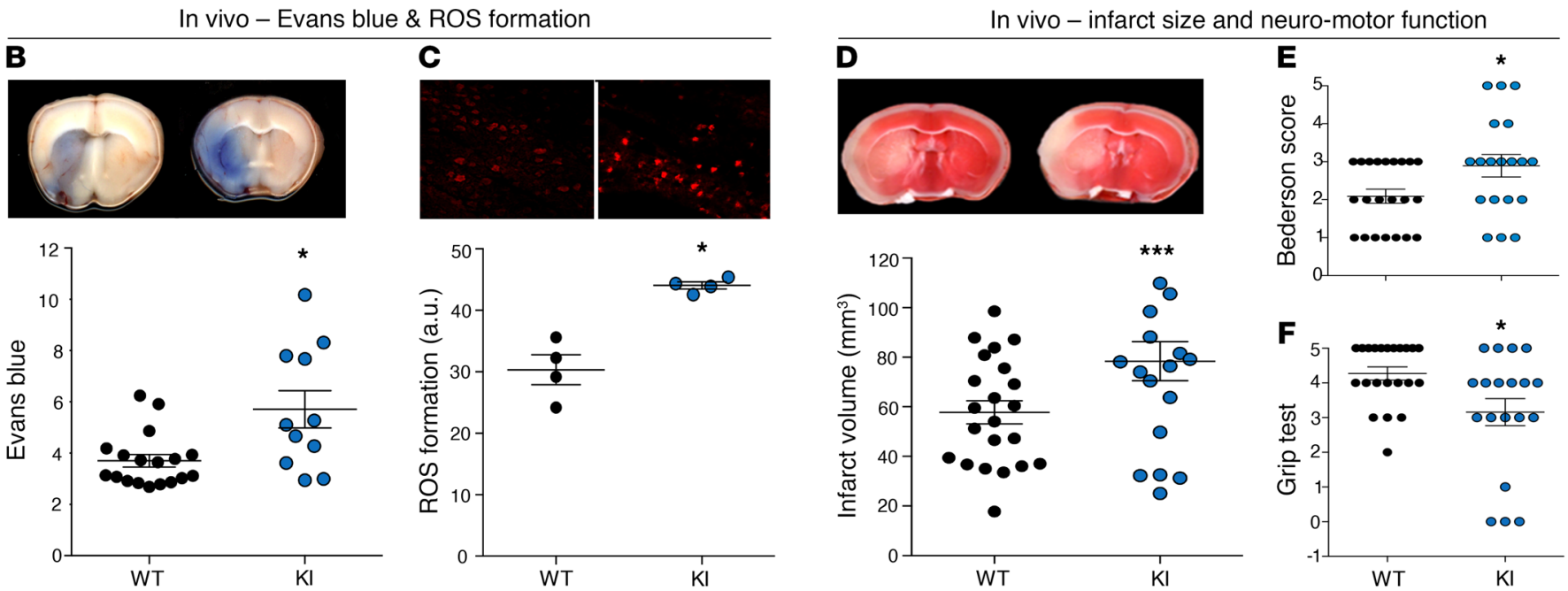

Figure 3. NOX5 leads to increased stroke size and BBB damage and aggravates neuromotor function. (A) In vivo, tMCAO was performed in WT and NOX5-KI mice. (B) Evans blue staining 24 hours after tMCAO showed BBB damage in both WT (black, $n=18$ ) and NOX5-KI (blue, $n=11$ ) mice. ${ }^{*} P<0.05$, 2-tailed unpaired $t$ test. (C) ROS production as measured by DHE staining 24 hours after stroke induction was increased in NOX5-KI (blue, $n=4,2$-tailed unpaired $t$ test) versus NOX5 WT (black, $n=4$ ) mice. ${ }^{*} P<0.05$. Original magnification, $\times 40$. (D) Increased infarct sizes are shown in NOX5-KI (blue, $\left.n=16\right)$ versus NOX5 WT mice (black, $n=21$ ). ${ }^{* * *} P<0.001$, 2-tailed unpaired $t$ test. Neurological behavior testing showed worse neurological functioning in NOX5-KI (blue, $n=19$ ) versus WT (black, $n=22$ ) mice, with worse $(\mathbf{E})$ Bederson and $(\mathbf{F})$ grip test scores. ${ }^{*} P<0.05$, 2-tailed unpaired $t$ test. Data are represented as mean \pm SEM in all experiments. Representative photographs are shown above the graphs.

To mimic human peripheral artery disease, we subjected NOX5-KI and WT mice to permanent ligation of the femoral artery, with angiogenesis being commonly used as a major histological outcome (27). Four weeks after occlusion, however, CD31 staining was comparable between WT and NOX5-KI mice (Supplemental Figure 3, A and B) as was blood flow (Supplemental Figure 3C).

To further investigate the role of NOX5 in MI and IR of the heart, we subjected adult NOX5-KI and WT mice to a permanent or transient occlusion of the left descending coronary artery. Again, 4 weeks after MI or IR, infarct sizes showed no differences between NOX5-KI and WT mice (Supplemental Figure 3D); 2 and 4 weeks after MI or after IR, ejection fraction, a common functional parameter, did not differ between NOX5-KI and WT mice (Supplemental Figure 3E). Finally, we examined cardiac contractility in response to increasing concentrations of the sympathomimetic dobutamine. NOX5-KI mice subjected to MI showed slightly enhanced contractility and relaxation. Upon IR, however, the response to dobutamine was not altered (Supplemental Figure 3F). Thus, in our humanized NOX5-KI mice, neither postischemic outcomes after permanent and/or transient ischemia of the heart nor long-term outcomes after HL were modified. These data suggest that the acute effects of NOX5 on ROS formation, infarct volume, and function are brain specific.

Acute postreoxygenation leakiness of human microvascular brain endothelial cells is prevented by pharmacological NOX5 inhibition.
To test the translatability of our findings into the clinical setting, i.e., pharmacological intervention, we used a primary culture of human brain microvascular endothelial cells (HBMECs) as an in vitro ischemia model (Figure 4A). Importantly, thrombectomy procedures have been recently associated with reperfusion damage and early BBB disruption, hemorrhagic transformation, and poor clinical outcomes. Thus, clinical trials have been designed to pharmacologically block the BBB opening in patients undergoing poststroke recanalization (4). Here, calcium is critical for tight junction function, particularly for cerebral microvessels and BBB stability, and poststroke calcium overload is a key mechanism of $\mathrm{BBB}$ disruption. Thus, we hypothesized that NOX5 is the missing mechanistic link in early BBB disruption and reperfusion injury. We subjected HBMECs expressing NOX5 endogenously to 6 hours of hypoxia, followed by 24 hours of reoxygenation and assessment of cell permeability (Figure 4B) in the presence or absence of the relatively NOX5-specific inhibitor ML090 (0.01 $\mu$ M; Supplemental Figure 4). Importantly, delayed induction of NOX4 also contributes to BBB disruption (17) and needed to be taken into consideration (Supplemental Figure 5). Hence, HBMECs were also treated both acutely and after reoxygenation with the relatively NOX4-specific inhibitor M13 $(0.2 \mu \mathrm{M})$ (Figure 4C). While the NOX4 inhibitor was protective when added either early or late, the NOX5 inhibitor was only protective when added early (Figure 4D). To finally test the possibility of a role for calcium-dependent 
A Human BBB model

B

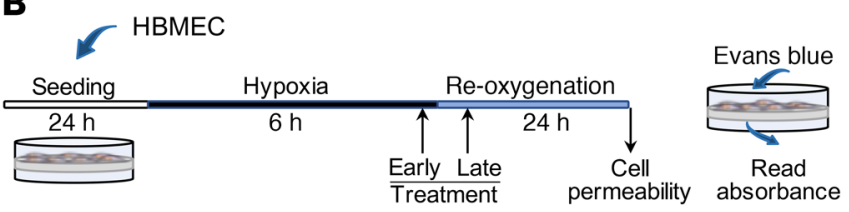

C

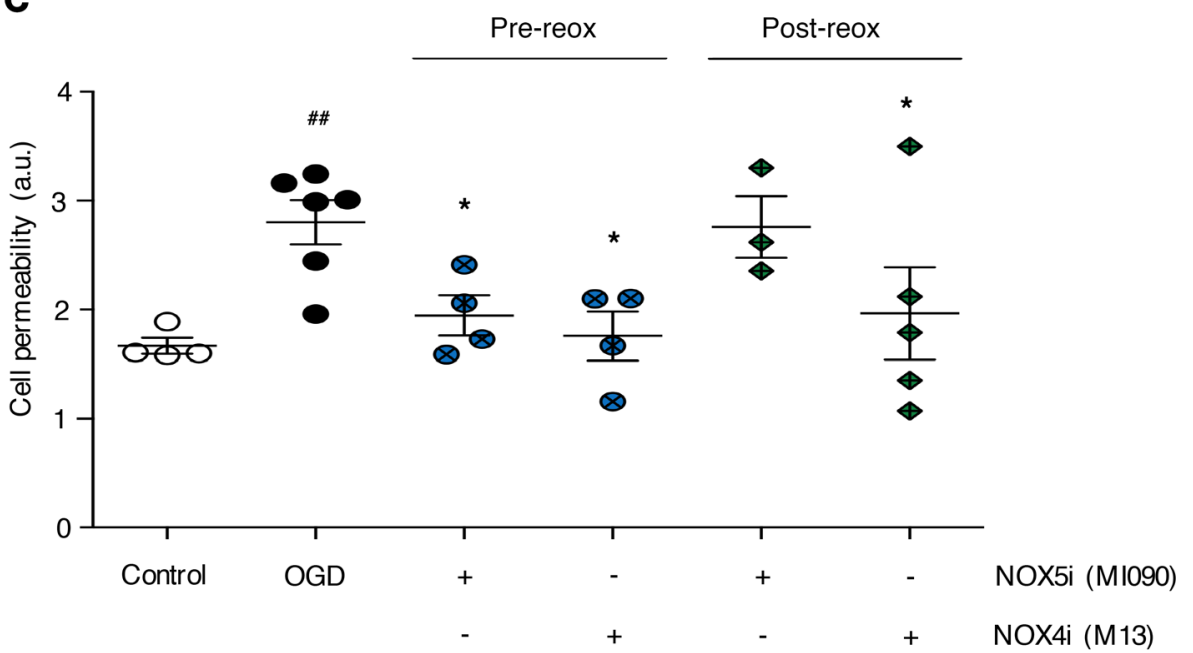

Figure 4. NOX5 inhibition before reoxygenation reduced cell permeability to basal levels using a human in vitro ischemia model. (A) HBMECs were seeded on Transwell inserts, where cell permeability was assessed using Evans blue dye. (B) After incubation and seeding under normoxic conditions, HBMECs were subjected to 6 hours of hypoxia and 24 hours of reoxygenation. Cells were treated with both NOX4 (M13) and NOX5 inhibitors (ML090) at early (20 minutes before reoxygenation) and late (20 minutes after reoxygenation) time points. (C) Cell permeability was assessed by measuring Evans blue fluorescence after hypoxia. Evans blue diffusion was significantly reduced in cells subjected to early treatment of MLO90 $(0.01 \mu \mathrm{M})$, which preferably inhibits NOX5, and M13 $(0.2 \mu \mathrm{M})$ which is mainly a NOX4 inhibitor ( ${ }^{*} P<0.05,2$-tailed unpaired $t$ test). In contrast, late treatment was only effective after M13 $(0.2 \mu \mathrm{M})$ treatment compared with that of nontreated cells $(\# \# P<0.01)$. Reox, reoxygenation. (D) NOX5 activation takes place within the first 30 minutes to 1 hour after hypoxia (see Figure 3), while NOX4 activation peak appears at around 5 hours after ischemia (20). Data are represented as mean \pm SEM in all experiments. NOX4i, NOX4 inhibitor.

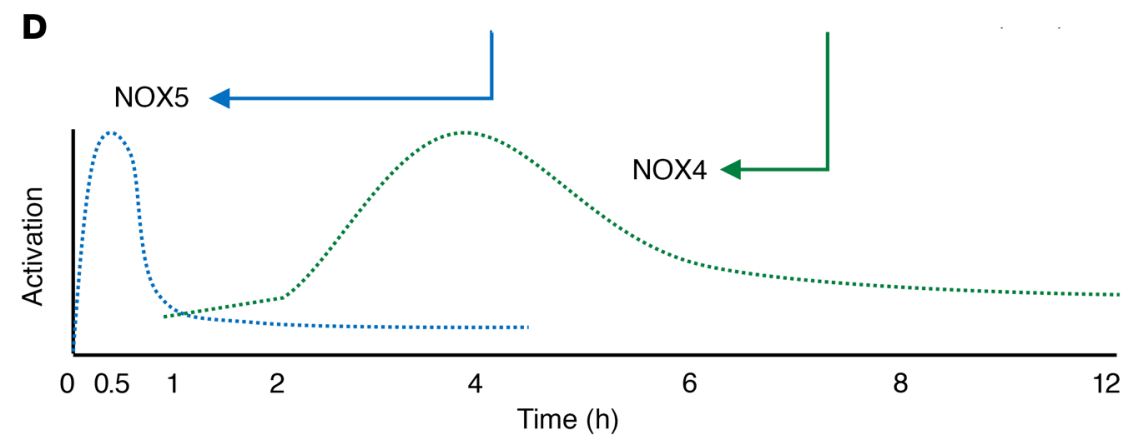

PKC and direct NOX2 activation (28), HBMECs were also treated before reoxygenation with the PKC inhibitor GF109203X (0.3 $\mu \mathrm{M})$, which, however, showed no effect, suggesting no relevant role of NOX2 (Supplemental Figure 6), in agreement with a previous in vivo preclinical randomized confirmatory trial (18). Thus, the role of calcium in acute BBB breakdown after reoxygenation/ reperfusion in stroke can be fully explained by NOX5, while NOX4 induction plays a key role in later stages. The efficacy of ML090 suggests a preventive pharmacotherapeutic option, ideally as a pretreatment or through coadministration with a thrombolytic drug, i.e., rtPA or a thrombectomy procedure.

Thus, we here identify NOX5-derived ROS as a missing link in acute stroke therapy between postreperfusion calcium surge, BBB breakdown, and worsened outcome. NADPH oxidases in general are promising therapeutic targets in ROS-associated diseases $(5,6,29)$. Since most preclinical validation experiments are conducted in mice and rats, which lack the NOX5 gene, NOX5 has been understudied. We provide what we believe is the first humanized NOX5-KI mouse model that mimics, to a large extent, the physiological pattern of NOX5 expression in humans, with substantial expression in endothelial cells and WBCs. In principle, our observed in vivo phenotype in NOX5-KI mice could thus derive from endothelial cells and WBCs, and we certainly cannot completely rule out a contribution of the latter. However, several lines of evidence support a fully sufficient role of endothelial NOX5. First, our in vitro mouse organotypic culture, essentially free of WBC contributions, and second, our HBMEC model, per definition entirely free of WBCs, are in full agreement with a crucial endothelial location of NOX5. Third, the detrimental role of NOX5 after reperfusion was highly specific for brain; NOX5 played no acute role in MI, transient heart ischemia, or $\mathrm{HL}$, suggesting indirectly the $\mathrm{BBB}$ and its cellular components as the main location of NOX5. Moreover, endothelial NOX5 would already be at the site of action with respect to BBB breakdown, whereas WBCs would need to adhere, and only a fraction of them would be preexposed to postreperfusion cal- 
cium surges. We also cannot exclude the possibility that NOX5 in other nonendothelial or non-WBC types may also contribute in vivo. From a clinical translational point of view, this may be a minor limitation because NOX5-directed pharmacotherapies, e.g., small molecules such as ML090, will always act systemically and not in a cell-specific manner.

Therapeutically, one additional aspect is worth considering, i.e., the previous identification of neuronal (and endothelial) NOX4 in stroke $(17,19)$. This enzyme does not contribute to the here-investigated early stages of BBB opening. Therefore, any prospective therapy will ideally want to cover both isoforms, acutely induced NOX5 and subacutely (i.e., within 4 to 6 hours) induced NOX4, to further inhibit the detrimental role of NOX4 and NOX5 at different stages of the pathomechanism.

Of direct clinical importance, our data suggest that thrombolysis and thrombectomy should be conducted in the presence of NOX inhibition to minimize the chances of a postreperfusion, calcium- and ROS-dependent acute and deleterious opening of the BBB. Our findings provide a clear rationale for further development of a pharmacological NOX $4 / 5$ inhibitor as a first-in-class neuroprotective strategy after stroke, to be coadministrated with the onset of reperfusion.

\section{Methods}

Details are provided in the Supplemental Methods.

Statistics. All data are expressed as mean \pm SEM. Statistical differences between mean continuous values were determined by Student's 2-tailed $t$ test. For repeated measurements, 2-way ANOVA was used.

Study approval. All animal experiments were performed according to the EU Directive 2010/63/EU for animal experiments, the German Animal Welfare Act, and Dutch law on animal experiments (Centrale
Commissie Dierproeven). Experiments were approved by the responsible authority of the Regierung von Oberbayern (Germany) and the Institutional Ethics Committee of the Universidad Autónoma de Madrid.

\section{Author contributions}

AIC, PWMK, and EG performed in vivo and in vitro experiments and data analysis and contributed to writing the manuscript. TA performed immunological phenotyping, which DHB, VGD, and MHDA supervised. JE helped with data acquisition, and FL helped with sample collection. HHHWS designed experiments, contributed to writing the manuscript, and acquired funding. MGL, CK, and JE acquired funding and methods. JE helped with some in vitro experiments and animal breeding.

\section{Acknowledgments}

This work was supported by the European Research Council, AdG RadMed 294683, an ERC PoC grant, SAVEBRAIN 139-101052, and H2020 project REPO-TRIAL (to HHHWS), the Spanish Ministry of Economy and Competence (SAF2015-63935R to MGL), the Deutsche Forschungsgemeinschaft (to $\mathrm{CK}$ ), the Fondo de Investigaciones Sanitarias (CP14/00008 and PI16/00735 to JE), European Cooperation in Science and Technology and Kootstra fellowships (to AIC), and the Bundesministerium für Bildung und Forschung Infrafrontier (01KX1012 to MHDA). A. BrounsStrzelecka, J. Debets, and H. van Essen are gratefully acknowledged for their expert technical support. We thank K. Wingler for help with generating and breeding of NOX5KI mice.

Address correspondence to: Harald H.H.W. Schmidt, Universiteitssingel 40, 6229 ER Maastricht, The Netherlands. Phone: 31.43.3881421; Email: h.schmidt@maastrichtuniversity.nl.
1. Radermacher KA, et al. Neuroprotection after stroke by targeting NOX4 as a source of oxidative stress. Antioxid Redox Signal. 2013;18(12):1418-1427.

2. Stankowski JN, Gupta R. Therapeutic targets for neuroprotection in acute ischemic stroke: lost in translation? Antioxid Redox Signal. 2011;14(10):1841-1851.

3. Behme D, Gondecki L, Fiethen S, Kowoll A, Mpotsaris A, Weber W. Complications of mechanical thrombectomy for acute ischemic stroke-a retrospective single-center study of 176 consecutive cases. Neuroradiology. 2014;56(6):467-476.

4. Warach S, Latour LL. Evidence of reperfusion injury, exacerbated by thrombolytic therapy, in human focal brain ischemia using a novel imaging marker of early blood-brain barrier disruption. Stroke. 2004;35(11 Suppl 1):2659-2661.

5. Kleikers PW, et al. NADPH oxidases as a source of oxidative stress and molecular target in ischemia/reperfusion injury. J Mol Med. 2012;90(12):1391-1406.

6. Casas AI, et al. Reactive oxygen-related diseases: therapeutic targets and emerging clinical indications. Antioxid Redox Signal. 2015;23(14):1171-1185.

7. BelAiba RS, et al. NOX5 variants are functionally active in endothelial cells. Free Radic Biol Med. 2007;42(4):446-459.
8. Manea A, et al. Human monocytes and macrophages express NADPH oxidase 5; a potential source of reactive oxygen species in atherosclerosis. Biochem Biophys Res Commun. 2015;461(1):172-179.

9. Jha JC, Watson AMD, Mathew G, de Vos LC, Jandeleit-Dahm K. The emerging role of NADPH oxidase NOX5 in vascular disease. Clin Sci. 2017;131(10):981-990.

10. Strengert M, Jennings R, Davanture S, Hayes P, Gabriel G, Knaus UG. Mucosal reactive oxygen species are required for antiviral response: role of Duox in influenza a virus infection. Antioxid Redox Signal. 2014;20(17):2695-2709.

11. Marzaioli V, et al. NOX5 and p22phox are 2 novel regulators of human monocytic differentiation into dendritic cells. Blood. 2017;130(15):1734-1745.

12. Shigemura T, et al. Superoxide-generating Nox $5 \alpha$ is functionally required for the human T-cell leukemia virus type 1-induced cell transformation phenotype. J Virol. 2015;89(17):9080-9089.

13. Takakura N, et al. Critical role of the TIE2 endothelial cell receptor in the development of definitive hematopoiesis. Immunity. 1998;9(5):677-686.

14. Tang Y, Harrington A, Yang X, Friesel RE, Liaw L. The contribution of the Tie2+ lineage to primitive and definitive hematopoietic cells. Genesis. 2010;48(9):563-567.

15. Furlan JC, Vergouwen MD, Fang J, Silver FL.
White blood cell count is an independent predictor of outcomes after acute ischaemic stroke. Eur J Neurol. 2014;21(2):215-222.

16. Grau AJ, et al. Leukocyte count as an independent predictor of recurrent ischemic events. Stroke. 2004;35(5):1147-1152.

17. Casas AI, et al. NOX4-dependent neuronal autotoxicity and BBB breakdown explain the superior sensitivity of the brain to ischemic damage. Proc Natl Acad Sci U S A. 2017;114(46):12315-12320.

18. Kleikers PW, et al. A combined pre-clinical meta-analysis and randomized confirmatory trial approach to improve data validity for therapeutic target validation. Sci Rep. 2015;5:13428.

19. Kleinschnitz C, et al. Post-stroke inhibition of induced NADPH oxidase type 4 prevents oxidative stress and neurodegeneration. PLOS Biol. 2010;8(9):e1000479.

20. McCann SK, Dusting GJ, Roulston CL. Early increase of Nox4 NADPH oxidase and superoxide generation following endothelin-1induced stroke in conscious rats. J Neurosci Res. 2008;86(11):2524-2534.

21. Jain AR, Bellolio MF, Stead LG. Treatment of hypertension in acute ischemic stroke. Curr Treat Options Neurol. 2009;11(2):120-125.

22. Matsushima S, Tsutsui H, Sadoshima J. Physiological and pathological functions of NADPH oxidases during myocardial ischemia-reperfu- 
sion. Trends Cardiovasc Med. 2014;24(5):202-205. 23. Wilkinson-Berka JL, Rana I, Armani R, Agrotis A. Reactive oxygen species, Nox and angiotensin II in angiogenesis: implications for retinopathy. Clin Sci. 2013;124(10):597-615.

24. Wilkinson-Berka JL, et al. NADPH oxidase, NOX1, mediates vascular injury in ischemic retinopathy. Antioxid Redox Signal. 2014;20(17):2726-2740.

25. Thallas-Bonke V, et al. Nox-4 deletion reduces oxidative stress and injury by PKC- $\alpha$-associated mechanisms in diabetic nephropathy. Physiol Rep. 2014;2(11):e12192.

26. Hahn NE, et al. NOX5 expression is increased in intramyocardial blood vessels and cardiomyocytes after acute myocardial infarction in humans. Am J Pathol. 2012;180(6):2222-2229.

27. Craige SM, et al. NADPH oxidase 4 promotes endothelial angiogenesis through endothelial nitric oxide synthase activation. Circulation. 2011;124(6):731-740.

28. Saul S, et al. A calcium-redox feedback loop controls human monocyte immune responses: The role of ORAI Ca2+ channels. Sci Signal. 2016;9(418):ra26.

29. Dao VT, et al. Pharmacology and clinical drug candidates in redox medicine. Antioxid Redox Signal. 2015;23(14):1113-1129. 